\title{
Machine Vision and Global Positioning System for Autonomous Robotic Navigation in Agriculture: A Review.
}

\author{
Reza Rahmadian ${ }^{1}$, Mahendra Widyartono ${ }^{2}$ \\ 1,2 Jurusan Teknik Elektro Fakultas Teknik Universitas Negeri Surabaya \\ UNESA Ketintang Surabaya \\ rahmadian.reza@live.com \\ mahendrawidyartono@unesa.ac.id
}

\begin{abstract}
Interest on robotic agriculture system has led to the development of agricultural robots that helps to improve the farming operation and increase the agriculture productivity. Much research has been conducted to increase the capability of the robot to assist agricultural operation, which leads to development of autonomous robot. This development provides a means of reducing agriculture's dependency on operators, workers, also reducing the inaccuracy caused by human errors. There are two important development components for autonomous navigation. The first component is Machine vision for guiding through the crops and the second component is GPS technology to guide the robot through the agricultural fields.
\end{abstract}

Index Terms - Machine Vision, GPS, navigation, Agriculture.

\section{INTRODUCTION}

Food security is one of the world's most serious problems. FAO[1] reports that in 2010-2012 the number of hungry people in the world remains unacceptably high with almost 870 million people chronically undernourished. This huge numbers has become an even more serious world problem as it involves not only the problem of the limited land available but also the growing population which results in a growing needs for food. Food shortages take place when food supplies are limited in the area and they can not provide the energy and nutrients needed by the people of the region. Lack of production is a major cause of food shortage, but political and environmental problems in food production become an important reason for it too. Today the emergence of dangerous politics of food scarcity has occurred, where some countries limit or ban exports and imports in an attempt to counter domestic food price rises. The food shortage increases the price, and continues not to fulfill the demands of a world with a population growing by 70 million people a year. In environmental problems, water shortage is the most immediate cause of food supply decrease. It is a serious matter that 70 percent of all water use is for irrigation [2]. The drilling of millions of irrigation wells in many countries, makes natural rainfall could not replenish renewable water supply. Besides that, other changes in weather and climate such as global warming or the rise of sea level will definitely be another threat to food security.

There are some cases in developing countries, for example Indonesia, where rice as the main food actually has been fulfilled by the national product, but the fact that the farming implements traditional techniques, causes inefficiency to happen in some ways, such as in spraying pesticide, harvesting, processing, transporting and storing the crops. This causes the crops' prices to become higher than they it should be. This inefficiency yields extra cost and consumption of time, which cause it to fail to attain its maximum productivity, and thus causes highly priced crops. Other crops such as soybean, corn, fruits and vegetables which potentially support food supply may experience similar problems. Recently even rice has to be imported in certain time of shortage showing the exceeding need for the food has become urgent and requires solution. In Japan and other developed countries some crops become too expensive to pick in peak harvesting season because of a shortage of workers, in part caused by an ageing population and/or the high wages cost of the workers. Some farmers must often leave the crops to rot says Wettels as in [1]. These developed countries have to give more opportunity for more efficient agricultural technology to be used in their crop management to anticipate food insecurity.

Based on the above reasoning precision agriculture needs to be developed to increase productivity and crop quality. Precision agriculture (PA) as Srinivasan in [3] defines it, is as a whole environmentally friendly strategy for farmers to use various farming methods which consider critical factors that effect yields. It is essentially a more precise form of farming management which makes 
use of modern technology. PA is the overall farm management used to optimize the crops produced while preserving resources. Agricultural technology is challenged to overcome the food shortage to supply food needs efficiently and in an evironmentally friendly manner.

Precision agriculture develops as it is supported by the progress of technology and management. Technology in agriculture benefits from advances such as those in agricultural production systems which have used the primarily developed technology for industries. They brought mechanization and synthesized fertilizers to agriculture and especially offered genetic engineering and automation which led to precision technology in agriculture[4].

The management makes use of the latest technologies such as satellite and information technology. Some countries such as Japan have initiated development of agricultural robots and techniques for autonomous navigation in the field . Now many institutions and manufacturers bring together their new technologies of autonomous navigation in agriculture[5]. Results of a number of researches on navigation have been released, and the most promising system for the future tends to focus on the combination of vision and Real Time Kinematics Differential Global Positioning System (RTK D-GPS).

The world's food requirements have become so high, and will continue to rise, so effective agricultural technology that is also environmentally friendly is certainly needed. With various characteristics of the fields used for farming; soil contour and type, weather, water, air, and plants, the variety of agricultural technologies should thus fit these characteristics.

Agricultural robots with sophisticated methods that produce the right tool for the farming need to be designed, tested, and produced. With the varied farming conditions of different agricultural countries, and disparities in their expertise, equipment, availability of electricity, funds and other resources, it is highly likely that manufacture of agricultural robots could increase effectiveness in a variety of different conditions. This thesis aims to provide guidance on the principles of the manufacture of agricultural robots using data and information gathered and analyzed from various sources and research results.

\section{AUTONOMOUS NAVIGATION}

Navigation is widely known as a methodology that guides vehicles from starting point to the final point. This method monitors the vehicle movement, determines the position of the vehicles, and controls the vehicle so that it will follow the direction as planned, and the final point of the vehicle would be achieved. As the time goes on, with the rapid development of technology, navigation nowadays can be done without the navigators present in the vehicles. Remotely monitoring the vehicle, the unmanned vehicle that is equipped with navigation sensors follows the desired path. This is one of the advantages of autonomous navigation.

In the beginning of research and development of autonomous vehicles, the main reason was to implement the vehicle to avoid situations that can be life threatening for humans, for example, the use of the vehicle in a high radiation area, to avoid direct contact with toxic substances, or to secure explosive materials. Recently, the development of autonomous navigation has enabled the possibilities for assisting people with their daily workload, such as in industrial zones. In car assembly, the autonomous navigation system steers arm robots to assist the assembly process. Gan-Mor et.al[6] said that autonomous system has been implemented on a Load-Haul-Dump truck, and successfully achieved full speed while operational in an underground mine. The other use for automatic navigation is automatic pilots, such as an unmanned spying drone or vehicle in battlefield for surveillance. One of the most widely used of the autonomous systems is the latest satellite navigation system, such as Global Positioning System (GPS) that has been implemented broadly to automatically guide these unmanned vehicles. The most important autonomous navigation system which assists people on a daily basis is the use of it to assist farmers in agricultural tasks. With integration of computer processing, machine vision and navigation systems, autonomous vehicles in agriculture and other industries are on the way to large scale commercialization.

\section{GLOBAL POSITIONING SYSTEM}

In Their paper, Subramanian, Burks, and Arroyo[7] described numerous literatures about autonomous and tele-operated vehicles. Murakami et.al in[7] stated that tele-operation has been used as guidance for operating an HST drive vehicle. However, tele-operation techniques have major challenges such as time delays in communication. Also, there is a requirement for full time human attention. That is why the use of GPS technology for vehicle guidance in agriculture can solve this problem. The rapid development of GPS-related technology has given the automation of open field procedure many possible benefits.

Global Positioning System is a satellite navigation system that can provide users with position and navigation. This system helps navigating naval vehicles to plot their course, and is commonly used for assisting users to navigate from one position to another. GPS satellites transmit signals to the receivers on the ground, although the receivers themselves do not transmit signal to GPS 
satellites. These satellites synchronize the operation and transmit signals to the receivers at the same time. There will be time delays between signals being sent and actually reaching receivers because each satellite has a different position and distance from the receivers. After estimating the distance from different satellites' transmissions, three dimensional calculations can be performed to determine the receiver's position. This can be achieved when it obtains transmitting signals from at least four different satellites.

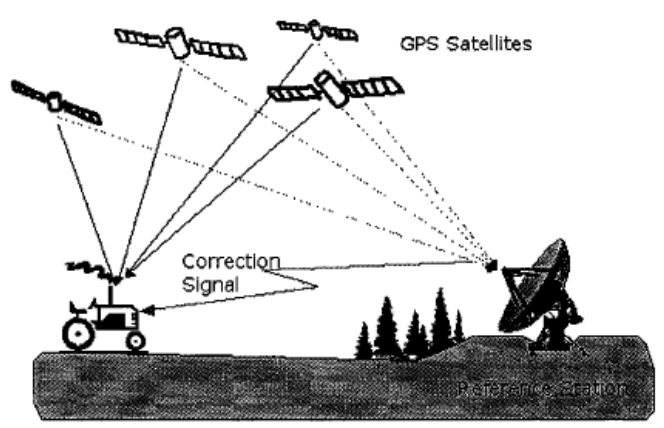

Figure 1. Principle of Differential Global Positioning System (DGPS).

Pseudoranging is one of the methods in GPS system to estimate the location of the receiver, Based on time differences, this method requires an accurate clock that records time, therefore atomic clocks are installed in the satellites and quartz clocks in the receivers. The GPS receivers continuously generate code and receive code from the satellites at the same time. The time required to synchronize these two codes is used to estimate distance between receivers and each of the satellites within range. Another method is the Carrier-phase; which is based on a detailed examination of the signals that satellites broadcast. GPS receivers will detect signals in the L-band and add them to a signal that is generated by the receiver; positional information can be derived from the observed frequency[8].

In developed countries, affordable prices for commercial sensors and processing GPS signals benefit most of the farmers [9], although they have limited function and accuracy, while it is a fact that a high-accuracy system for GPS-related technology comes at an expensive cost. Fortunately, there is a GPS system called Differential-GPS (DGPS) that is already widely used, and which has a lower cost. This system, when used in agricultural operations such as automation guidance, and application of fertilizer or sprays, has relatively low expenses [10];[11]. The use of GPS systems, especially Differential GPS has proven beneficial for agriculture in general.

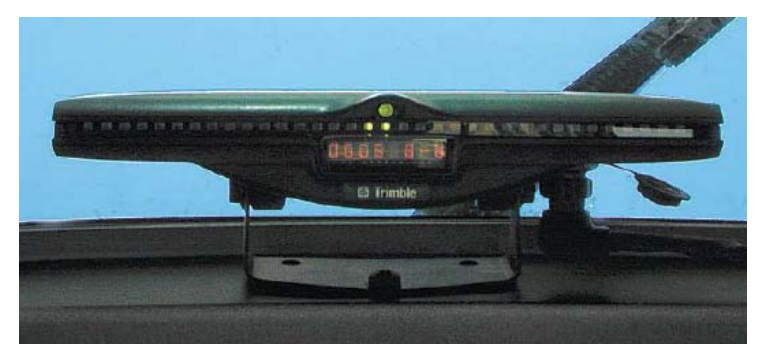

Figure 2. LED bar mounted on top of a tractor that indicate if the GPS unit is on track, Green LED means the tractor is on track.

Some studies show that GPS systems have been developed widely in agricultural navigation. Gan-Mor et.al.[12] states that recent technology called S-DGPS can achieve accuracy to approximately $10 \mathrm{~cm}$ using the same remote ground station that was used in earlier DGPS. In the same area of study, O'Connor et.al.[13] a researcher from Stanford University developed a linear vehicle model to control a John Deere Model 7800 tractor using Carrier-Phase Differential GPS along four 50 $\mathrm{m}$ straight row courses, each of which had an antenna carrier-phase GPS system at the end. The result achieved from the first experiment for the mean tracking error was less than $1 \mathrm{~cm}$ and the Standard Deviation (S.D) of the GPS was approximately $2 \mathrm{~cm}$. This is considered accurate when compared to the control value for tracking error, which was less than $5 \mathrm{~cm}$, and the S.D of the GPS, which was less than $10 \mathrm{~cm}$.

\section{MACHINE VISION}

Machine vision is a technology and method to automatically capture images or digital images, process and analyze the images to make decisions and pass the result to the automatic controller. Machine vision technology is not only able to capture visible objects in its color region, but it is also able to capture light with longer wavelength than visible light, such as Infrared and Ultraviolet. When these invisible to human eye - lights are reflected from objects and captured by machine vision, the important information received can be very useful in several ways. For example, in harvesting fruits, the information from these lights could determine the fruit's maturity, and the kinds of disease and stress level that the fruits may have. For other plants, like vegetables, the information also can be useful in determining plant and vegetables variety, ripeness, and quality [14].

A simple machine vision system has several common features such as Image acquisition, preprocessing, feature extraction and detection or segmentation, as seen in Figure 5. 


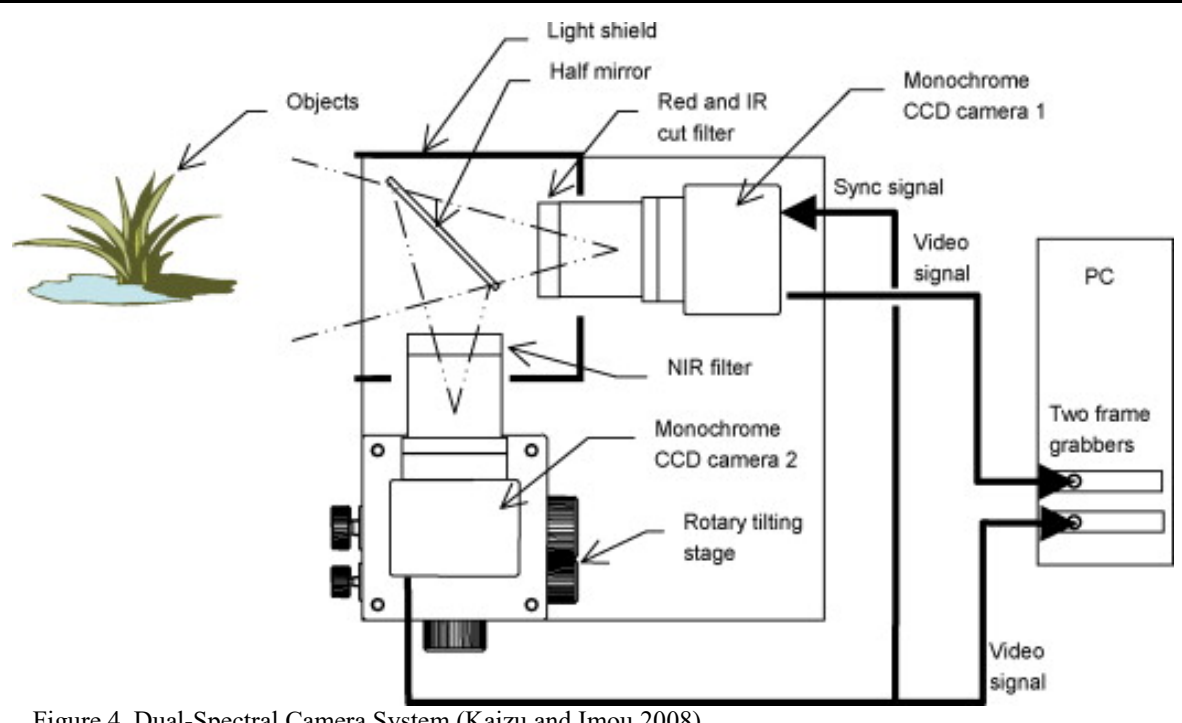

Figure 4. Dual-Spectral Camera System (Kaizu and Imou 2008).

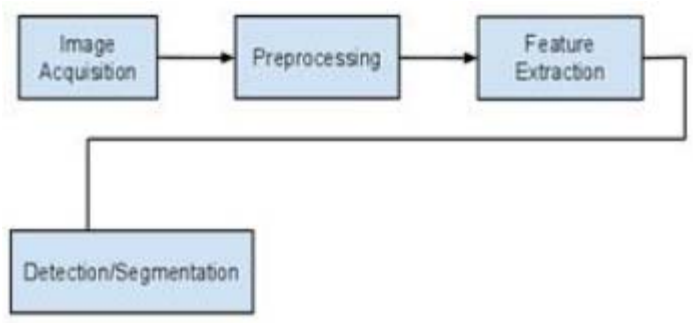

Figure 3. Simple Machine vision system (Segerbland and Delight, 2012).

Image acquisition involves capturing images of the environment; devices such as cameras are one example. After acquiring images, a preprocessing method will process the images, removing some noise using removal algorithm or filtering algorithms, and/or enhance them. Feature Extraction will retrieve desired properties of the images such as shapes, and send them to Detection or segmentation process. In here, the images properties will be sorted and decide which properties are relevant for further processing[15].

Imaging the object with machine vision methodology can be done in several different ways, such as monochrome and color imaging.

Monochrome imaging means only one color or shade of one color is captured

Image acquisition involves capturing images of the environment; devices such as cameras are one example. After acquiring images, a preprocessing method will process the images, removing some noise using removal algorithm or filtering algorithms, and/or enhance them. Feature Extraction will retrieve desired properties of the images such as shapes, and send them to Detection or segmentation process. In here, the images properties will be sorted and decide which properties are relevant for further processing[15].

Imaging the object with machine vision methodology can be done in several different ways, such as monochrome and color imaging. Monochrome imaging means only one color or shade of one color is captured from the object, or in this method, it captures the visible light reflection from the object. Whereas color imaging captures three basic visible colors in human eyes, red, green, and blue (RGB). Images that have been captured need to be processed and analyzed to extract the desired information. Techniques such as image enhancement are commonly used to correct problems that appears in digital images such as poor contrast or noise [16]. By adding filter and pixel-topixel operation, image enhancement is able to correct inconsistencies in the captured image caused by non uniform illumination. Once the problem has been corrected, analyzing software such as fuzzy or neural networks will extract the desired information.

The advantages of using machine vision for sensing are that it is highly accurate, and deliver consistent results. This will improve productivity in any industry and so can lead to higher quality product and greater cost efficiency. Machine vision in the agricultural sector has been used in various applications, such as precision farming, land-based and aerial-based remote sensing, and post-harvest technology due to its ability to recognize size, color and shape of objects, as well as its ability to provide numerical attributes of the imaged object itself[17].

The image captured by the camera is actually a light reflection from the object, and a device called a charge-coupled device (CCD) changes the reflected light to electrical signals. For monochrome imaging, there is only a single-chip CCD and it is capable of detecting visible light and near infrared (NIR) light which is invisible to human eyes. Monochrome cameras have been used to capture images for aiding vehicle navigation. Dickmanns and Mysliwetz[18], conducted several studies using monochrome cameras mounted to a 5-ton van, to 


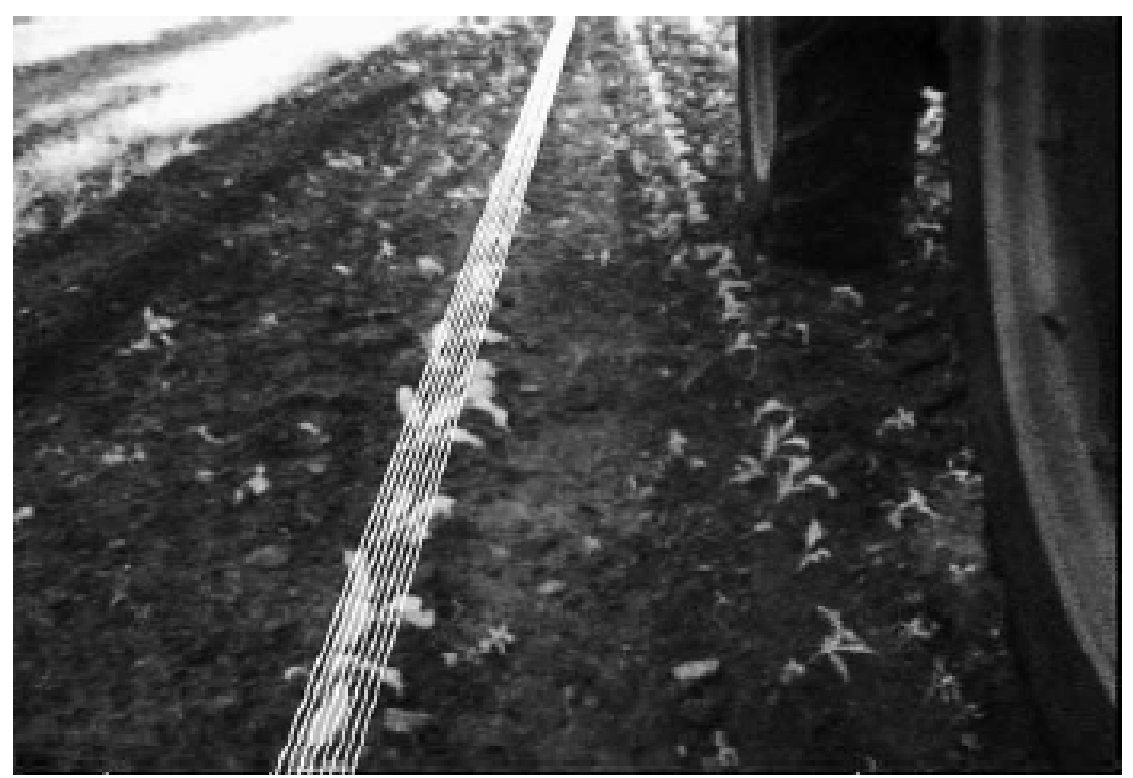

Figure 5. The detected plant row Åsrand and Baerveldt[29].

recognize both horizontal and vertical road curvature parameters. Matthies et.al.[19], also used a pair of monochrome cameras in research to detect obstacles under several conditions such as lowvisibility, and night time. The research proved that, machine vision using a pair of monochrome cameras was able to provide a usable range of data for unmanned ground vehicles relatively costeffectively. Several researches used color CCD camera for their device to capture the image, and by alternating the pixel in the camera's CCD, a single chip of CCD can also be used for color imaging. However, using only single-chip CCD may not be appropriate for image processing in machine vision. A camera system with three-chip CCD may be more suitable for image processing, because the image it captures has a near true color or almost the same color density as the object. Several study used color camera as their imaging device, like Jiang et.al. [20] which used a KOKO color CCD camera and a FCBEX780S single CCD analog color video camera.

A Sony DCR-PC10 digital video camera mounted on a six-row automatic rice transplanter was used in an experiment investigating machine vision based guidance systems. The experiment was conducted by Chen et.al.[21] and showed that the system was able to analyze captured images and tell the difference between the border of the rice field and concrete or soil banks, and rows of rice seedlings. It was also capable of detecting the seedlings rows until the edge of the field. The detection accuracy recorded for concrete bank was $99.2 \%$, for soil bank $98.2 \%$, and for the rice seedlings $98.9 \%$.

Xue et.al.[22] used camera arrangement techniques called fixed forward field of view (far FOV) for developing an autonomous robot for agricultural field applications. This camera placement worked well for tall and mature plants, encountered problems with small plants, and was insufficient for turning the robot at the end of the crop row. Therefore, the researchers applied two methods into the far FOV. The first one was "near FOV" for detecting small or short plants and the other method was "lateral FOV" for turning the robot at the end of the crop row. The result showed that the far FOV guidance had achieved a successful experiment, with an average error of $1 \mathrm{~mm}$ and a standard deviation of $7.1 \mathrm{~mm}$, in which the worst case guidance error was $15.8 \mathrm{~mm}$. In addition, no plants were touched or run over during the tests. Xue et.al.[22] also stated that a similar study had been done previously by Chen et.al.[21], Bak and Jakobsen [23], and Xue and $\mathrm{Xu}$ [24], applying the same method in the development of autonomous robots for field applications.

Another method for piloting a vehicle or robot in agricultural fields using vision techniques requires crop rows for navigational assistance. A machine vision-based automatic row guidance approach research was able to navigate a horticultural robot at a speed of $1.8 \mathrm{~km} / \mathrm{h}$ and attain an accuracy of about $15 \mathrm{~mm}$, using crop rows as a navigational aid[25]. Slaughter et.al.[26] used two cameras for monitoring two separated crop rows $3 \mathrm{~m}$ apart, in order to provide more accuracy when there were missing plants in the crop rows. The vehicle had been tested in commercial field at a travel speed of $16 \mathrm{~km} / \mathrm{h}$, and had RMS position error from 4.2 $\mathrm{mm}$ to $12 \mathrm{~mm}$. Kise et.al.[27] in their research used a stereovision-based method to detect crop rows in 
soya beans field. The core element in this research was a stereovision camera, that capture images based on two planes images that simultaneously taken with a binocular lenses inside the camera. The research investigated the fundamental technology for agricultural machinery navigation using stereovision-based system. Field tests showed that the system provided reliable and accurate tractor or vehicle navigation information for crop-row detection. The tractor in this experiment was able to achieve RMS error of lateral deviation less than 5 $\mathrm{cm}$, when it was guided automatically using a stereovision navigation system, following both straight and curved rows at an average speed of 3 $\mathrm{m} / \mathrm{s}$. In addition, Kaizu and Imou[28] developed a method called dual-spectral camera system that used a pair of monochrome cameras (Figure 6). This method was used to detect rows of rice seedlings to aid the navigation of rice transplanters, through rice field that had strong light reflection due to the water surface. The field experiment recorded that this method was able to detect rice seedling rows, and to eliminate noise obtained from reflection of the sky and tree crown. This experiment was conducted under cloudy conditions. By obtaining row guidance information from five wheat rows in a plan view of the field, and using analysis of the periodic NIR intensity, Hague and Tillett[25] in their research had acquired RMS position error of $15.6 \mathrm{~mm}$ at a travel speed of $5.8 \mathrm{~km} / \mathrm{h}$.

An experiment on two commercial machine vision guidance systems was conducted by Sogaard et.al., for beets and rape crop, and recorded standard deviation for guidance ranging from $11-22 \mathrm{~mm}$ at travel speeds from 5 to $10 \mathrm{~km} / \mathrm{h}$. Another vision based row-following system research that was done by Åsrand and Baerveldt[29], resulted a standard error between 6 and $12 \mathrm{~mm}$ depending on the crop size which varied from 5 to $30 \mathrm{~cm}$, Figure 7 shows the row detection for the experiment. Two types of agricultural vehicles that were implemented with this system were a row cultivator and an agricultural mobile robot. A new method of Hough transform was presented in their study for more robust crop row recognition.

Machine vision techniques based on Hough transform are commonly applied to identify crop rows. Hough transform is able to efficiently detect discontinuous lines or curves in picture, which in machine vision crop rows are translated as continuous lines. Marchant et.al.[30] conducted an experiment on of autonomous vision-guided vehicles, using a row following method in transplanted cauliflowers fields. The vehicle travelled at the speed of $0.7 \mathrm{~m} / \mathrm{s}$ along the $70 \mathrm{~m}$ long field. Hough transform is used in this experiment, not only to detect crop rows but also to deal with various degrees of disruptions such as missing plants or the presence of weeds. These disruptions were randomly positioned on the field by the researchers.
The result recorded in this study shows the overall standard deviation of the vehicle was $20 \mathrm{~mm}$, while the accuracy of vehicle position was within about 8 $\mathrm{mm}$.

Mas et.al. also used Hough transform to identify crop rows in a soybean field. This study was conducted to obtain and mark the center of the path that an automatic guidance tractor had to follow later. The result showed that Hough transform was able to effectively overcome noise problems associated real crop images, and it was capable of providing adequate filtering to identify crop rows. In the same field, Jiang et.al.[20] used Hough transform algorithm in their research on machine vision based crop-row detection robots, to detect multi crop rows in different light conditions. The experiment showed that the Hough Transform algorithm was able to detect all crop centerlines under different weather and light conditions. Hough transform and its developments are able to efficiently detect crop rows, and they deal with various degrees of disruptions under different weather or different light conditions.

Machine vision has been widely used in the agricultural area, with several researches recording that it has high accuracy for navigating agricultural vehicles. The row-following method is one of the machine-vision based navigation systems that is commonly used. The implementation of algorithm in this method shows that the accuracy achievable is sub-centimeter based. This ability to improve productivity in any industry can lead to high quality product and cost efficiency. However, several conditions for applying machine-based navigation need to be considered. For example in the case of a planned agricultural field, some fields may not have crop row planning, or they have poor planning, and these conditions may affect the operating speed of the agricultural vehicle and may break the existing crops. Another condition is natural lighting; machine vision needs to capture images of the object and process the information. Low or excessive lighting conditions can result in low quality or high noise images, which can give error reading in image processing. In addition, reflection from the surface of water in paddy fields could also mislead image processing, because the reflection on the surface of the water will be considered as crop rows by the machine vision system. Future research has to be performed to overcome these conditions.

\section{CONCLUSION}

The pace of Robot development has been so fast; it has become an important topic of research for universities. Companies compete with each other to produce the latest and most advanced robots. Robots are so widely used that it seems a robot may the human in tasks in almost every part of human's life, from difficult, and dangerous tasks, to repetitive and simple tasks at home. In agriculture, 
autonomous robots replace humans in tasks precisely by reducing the weakness which humans usually bring to work. Robots especially facilitate the work of farmers in order to increase productivity and quality of agricultural products.

Precision agriculture also depends on satelite positioning systems like GPS to locate the precise position of crops in a field. GPS as the satellite navigation system in precision agriculture for automatic guidance is an effective tool for autonomous vehicles because GPS helps steer the vehicle around the field. With its integration with several sensors and actuators such as laser, ultrasonic or machine vision, GPS will provide a path or a data map that will navigate an agricultural vehicle between the crops with precision. GPS satellites synchronize the operation and transmit signals to the receivers on the ground, while its receivers continuously generate and receive code from the satellites at the same time. GPS systems have been developed widely in agricultural navigation; researchers have improved the technology to agree with certain field needs. Although Differential-GPS (DGPS) with usual GPS similar capabilities has proven to be cheaper, accuracy has become the main aim of applying GPS in agricultural robots. Systems like S-DGPS and RTK-GPS have also proved to be more accurate when they are mounted on autonomous robots to navigate. GPS has proven to be more effective in terms of time delay and the requirement of full time human attention needed compared to similar methods of other tele-operation for communication. GPS automation has thus given an open field procedure of many possible advantages.

Another technology that is used in precision agriculture is machine vision as the integral system in autonomous navigation vehicle. Machine vision has various types and applications in the agricultural sector. Based on its task to capture the object in the field, the use of the camera becomes the important part that always obtains modification by the researchers. The monochrome camera (single or dual spectral camera) is one of the cameras that are used to capture images for aiding vehicle navigation, similar to Sony DCR-PC10 digital video cameras which are mounted on a six-row automatic rice transplanter used for its navigation system. A stereovision camera with binocular lenses inside the camera is also a successful technology for agricultural machinery navigation systems. A camera system with three-chip CCD that is KOKO color CCD camera and a FCB-EX780S single CCD analog color video camera is more suitable for image processing. And other machine vision technology is the camera arrangement techniques called fixed forward field of view (far FOV: near and lateral FOV) for developing an autonomous field agricultural robot. Besides that, of course the commonly applied machine vision techniques based on Hough (1962) transform is applied to identify crop rows in various conditions.

\section{REFERENCES}

[1] FAO, WFP and IFAD. 2012. The State of Food Insecurity in the World 2012. Rome, FAO.

[2] Brown, Lester. 2009. Worldwide Crisis: The Geopolitics of Food Scarcity. Spiegel Online.

[3] Kumar, S., and C. S. Sudeep. "Robot for Precision Agriculture". 1 $3^{\text {th }}$ National Conference on Mechanism and Machines (2007).

[4] Gan-Mor, Samuel, et al. "Guidance of Autonomous Vehicle for Greenhouse Transportation”. ACTA Horticulture, no 443 (1997): 99-104.

[5] Torii, Toru. "Research in Autonomous Agriculture Vehicles in Japan". Computer and Electronics in Agriculture 25, (2000): 133-153.

[6] Gan-Mor, Samuel, et al. "DGPS Tractor Guidance : Implement Guidance Error as Affected by Field Conditions using Automatic". American Society of Agricultural Engineers Paper, no 021153 (2002).

[7] Subramanian, A., T. F. Burks, and A. A. Aroyyo. "Development of Machine Vision and Laser Radar Based Autonomous Vehicle Guidance Systems for Citrus Grove Navigation". Computers and Electronics in Agriculture 53 (2006): 130-143.

[8] Paul, Andre. "Design of An Autonomous Navigation System for A Mobile Robot". Master Thesis., University of McGill, Montreal, 2005.

[9] Zhang, N., M. Wang, and N. Wang. "Precision AgricultureA World Wide Overview". Computers and Electronics in Agriculture 36 (2002): 113-132.

[10]Abidine, Aziz Z., et al. "Application of RTK GPS Based Auto Guidance System in Agricultural Production". The American Society of Agricultural and Biological Engineers Paper, no 021152 (2002).

[11]Gan-Mor, Samuel, et al. "DGPS Tractor Guidance : Implement Guidance Error as Affected by Field Conditions using Automatic". American Society of Agricultural Engineers Paper, no 021153 (2002).

[12]Gan-Mor, Samuel; Bruce L. Upchurch and Rex L. Clark. "GPS Tractor Guidance : Implement Laterat Position Accuracy Inder RTK". Computer and Electronics in Agriculture, no 59 (2007): 31-38.

[13]M. O'Connor et al, 'Tractor drivers soon may say, " Look, Ma! No hands!' ', Stanford Report 1997. [Online]. Available: http://news.stanford.edu/news/1997/september24/gsbtractor. html. [Accessed: 02- Jun- 2011].

[14]Yang, Linghe. "A Fruit Recognition Method for Automatic Harvesting". Master Thesis., University of Windsor, Canada, 2007.

[15]E. Segerblad, and Bjorn Delight 'Machine Vision in Agricultural Robotics - A short overview", IDT Workshop on Interesting Results in Computer Science and Engineering. [Online]. Available: http://www.idt.mdh.se/kurser/ct3340/ht11/MINICONFERE NCE/FinalPapers/ircse11_submission_2.pdf. [Accessed: 10Jun- 2011].

[16]De-An, Z., L. Jidong, J. Wei, Z. Ying, and C. Yu. "Design and Control of an Apple Harvesting obot". Biosystem Engineering 110, (2011): 112-122.

[17] Lu, J., D. Zhao, W. Ji, Y. Chen, and H. Shen. "Design and Research on Vision System of Apple Harvesting Robot". Third International Conference on Intelligent HumanMachine Systems and Cybernetics IEEE 1, (2011): 177-180.

[18]E.D. Dickmanns and B. Mysliwetz: Recursive 3-D Road and Relative Ego-State Recognition. IEEE-Transactions PAMI, Vol. 14, No. 2, Special Issue on 'Interpretation of 3-D Scenes', Feb 1992, pp 199-213.

[19]C.F. Olson.., L.H Matthies, M. Schoppers, and M.W. Maimone. "Stereo ego-motion improvements for robust rover navigation". Robotics and Automation. Procedings 2001 ICRA. IEEE international confrence, (2001). 
[20]T. Junhua., H. Jiang, and W. Zhou. "Development of Automoatic System for the Seedling Trasplanter Based on Machine Vision Technology" College of Biosystems Engineering and Food Science. Zhenjiang University, (2010).

[21]Zhao, C. W. Huang, L. Chen, Z. Meng, Y. Wang, and F. Xu. "Harvest Area Measurement System Based on Ultrasonic Sensors and DGPS for Yield Map Correction". Precision Agric 11, (2010): 163-180.

[22].Xue, J., L. Zhang, T.E. Grift. "Variable field-pf-view machine vision based row guidance of an agricultural robot.". Computer and Electronics in Agriculture, no. 84 (2012) 8591.

[23]Bak, T., H. Jakobsen. "Agricultural robotic platform with four wheel steering for weed detection. Biosyst. Eng, 87 (2) $125-136$.

[24] Xue, J., L. Xu. "Autonomous agricultural robot and its row guidance". 2010 International conference on Measuring Technology and Mechatronics Automation, 1 (2010) 725729.

[25]Hague, T., J. A. Marchant, and N. D. Tillett. "Ground Based Sensing Systems for Autonomous Agriclutural Vehicles". Computers and Electronics in Agriculture 25 (2000): 11-28.

[26] Harrel, R. C., P. D. Adsit, R. D. Munilla and D. C. Slaughter. "Robotic Picking Citrus". Robotica 8, no 4 (1990): 269-278.

[27] Kise, M., N. Noguchi, K. Ishii, and H. Terao. "Field Automation Using Robot Tractor". Proceedings of the Automation Technology for Off-road Equipment, (2002).

[28] Kaizu, Y., and K. Imou. "A Dual-Spectral camera system for paddy rice seedling row detection". Computers and Electronics in Agriculture, 63 (2008). 49-56.

[29] Astrand, B., and A. Baerveldt. "A vision based rowfollowing system for agricultural filed machinery". Mechatronics, 15 (2005). $251-269$.

[30] Hague, T., J. A. Marchant, and N. D. Tillett. "Ground Based Sensing Systems for Autonomous Agriclutural Vehicles". Computers and Electronics in Agriculture 25 (2000):11-28 